\title{
Interactive comment on "Reanalysis
} intercomparison of potential vorticity and potential-vorticity-based diagnostics" by Luis F. \section{Millan et al.}

\section{Luis F. Millan et al.}

luis.f.millan@jpl.nasa.gov

Received and published: 26 February 2021

\section{See attached}

Please also note the supplement to this comment:

https://acp.copernicus.org/preprints/acp-2020-1181/acp-2020-1181-AC1-

supplement.pdf 